\title{
Caracterização \\ Físico-Química e Análise Sensorial de Presuntos com Redução no Teor de Sódio
}

\author{
Júlie E. S. Carneiro, Leonardo M. Calixto, Murillo S. M. Monteiro, \\ Tatianne F. de Oliveira \& Michelly F. M. Martin
}

O efeito da substituição do cloreto de sódio por cloreto de potássio sobre a qualidade de presuntos reestruturados foi investigado nas influências nas características físicoquímicas e sensoriais do produto. Para produção do presunto, usou-se pernil desossado sem gordura superficial. Foram preparadas 4 formulações de presunto (F1, F2 e F3 reduziu-se, respectivamente, $15 \%, 30 \%$ e $45 \%$ ) no qualem que foram submetidos ao processo de cura seca. Com $45 \%$ de substituição, aumentou-se o teor de cinzas e carboidratos. Observou-se que ao aumentar a concentração do cloreto de sódio, houve redução do pH e capacidade de retenção de água.

Palavras-chave. presunto; cloreto de potássio; cloreto de sódio.

The effect of the replacement of sodium chloride by potassium chloride on restructured hams quality was investigated in influences on the physical-chemical and sensorial characteristics of the product. For the production of ham, boned shank with no surface fat was used. Four formulations of ham (F1, F2 and F3 reduced, respectively, $15 \%, 30 \%$ and $45 \%$ ) were prepared in which they were subjected to the dry curing process. With $45 \%$ replacement the content of ashes and carbohydrates was increased. It was observed that by increasing the concentration of sodium chloride there was a reduction of $\mathrm{pH}$ and water retention capacity.

Keyword. ham; potassium chloride; sodium chloride. 


\section{Introdução}

As carnes são alimentos perecíveis e apresentam vida de prateleria curta em função das condições de armazenamento. Desde a antiguidade, o homem sempre buscou preservar as características e a qualidade destas, com o desenvolvimento e a conservação da espécie contribuiram para o surgimento de processos e tecnologias de transformação, inicialmente, eram bastantes rudimentares, porém foram os precursores para os altos padrões tecnológicos da atualidade para preservar o alimento. A fabricação de produtos cárneos cozidos é uma das tecnologias que visa aumentar a vida de prateleira das carnes e diversificar a oferta de derivados, como presunto, apresuntados, salchicha dentre outros ${ }^{1}$.

Ao mesmo tempo que as tecnologias vieram para melhorar a qualidade de vida do ser humano, também trouxeram várias consequências negativas. Uma das consequências ruins ao ser humano é alta ingestão do sódio que pode acarretar sérias doenças, tais como a hipertensão e insuficiência cardíaca, insuficiência renal dentre outras. Calculou-se que, só nos EUA, os benefícios de saúde potenciais de uma redução de $3 \mathrm{~g} /$ dia de sal iria reduzir o número de mortes em casos de 44-92.000 e economizar cerca de U\$ 10 bilhões. Da mesma forma, estima-se que reduzindo a quantidade de sal ingerido a $6 \mathrm{~g} /$ dia seriam impedidas 17.500 mortes por ano no Reino Unido $^{2}$. Da mesma forma, estima-se que a redução da quantidade de sal ingerida a $6 \mathrm{~g} /$ dia impediria 17.500 mortes por ano no Reino Unido ${ }^{3}$. Por estas razões, os órgãos internacionais estabeleceram metas para uma redução no consumo de sódio até $2 \mathrm{~g} / \mathrm{dia}$.

Estima-se que cerca de $70 \%$ do sódio consumido pelo homem é representado pelos produtos industrializados, tais como carnes processadas (18\%), pão e pastel (13\%), produtos lácteos $(12 \%)$, molhos e prontos $(11 \%)$, e fast foods $(16 \%)^{4,5}$. E $30 \%$ representam a quantidade de sódio por parte dos consumidores em casa. A redução do cloreto de sódio nos produtos cárneos representa cerca de $1 / 4$ da ingestão total de sódio. A redução deste é uma alternativa para a minimização de doenças, consequentemente, melhorar a qualidade de vida dos indivíduos ${ }^{1,3,6}$.
O cloreto de sódio é adicionado nos produtos cárneos para aumentar a vida de prateleira e palatabilidade, devido à sua importância na formulação deste, sua redução pode afetar de forma negativa sua qualidade geral. Quando se trata de qualidade sensorial, cloreto de sódio contribui para a salinidade e o sabor global, ao mesmo tempo, a supressão da amargura. Assim, o desenvolvimento de produtos com redução de sódio palatáveis é importante, a fim de orientar os consumidores para escolhas alimentares mais saudáveis. A substituição parcial do $\mathrm{NaCl}$ por $\mathrm{KCl}$ tem mostrado ser uma das melhores alternativas para a redução do teor de sódio. Estudos têm mostrado que $\mathrm{KCl}$ induz um gosto amargo em concentrações elevadas, em lombos curado a seco, salsicha fermentado e do presunto cozido. Esses resultados mostram que o nível máximo de substituição de $\mathrm{KCl}$ pode variar entre diferentes tipos de produto $^{7,8}$.

A necessidade de desenvolvimento de produtos com redução no teor de sódio devido à crescente conscientização do consumidor com relação ao consumo de alimentos mais saudáveis, assim como a demanda da indústria por produtos que ao mesmo tempo que tenham reduzido teor de sódio apresentem características físico-químicas e sensoriais às quais o consumidor já está habituado, justificam este estudo.

Portanto, o objetivo deste estudo foi elaborar um presunto com o menor teor de sódio possível em relação aos produzidos no Brasil e investigar as influências da substituição do cloreto de sódio nas características físicoquímicas e sensoriais do produto.

\section{Metodologia}

\section{PROCESSAMENTO DE PRESUNTO}

Para cada uma das três repetições, pernil de porco desossado e sem gordura, foram comprados em comércio local (Goiânia, Goiás, Brasil). Foram preparadas 4 formulações de presunto (Tabela 1) em 3 lotes distintos. Na fórmula controle $(\mathrm{C})$, não houve redução no teor de sódio por cloreto de potássio. Porém, nas formulações F1, F2 e F3, reduziu-se, respectivamente, $15 \%, 30 \%$ e $45 \%$.

Os pedaços cárneos foram submetidos ao processo de 
cura seca, com a adição dos ingredientes na superfície por fricção. Em seguida, foi acondicionada sob refrigeração a $277 \mathrm{~K}$ por 2,5 dias em sacos plásticos de polietileno de baixa densidade. Após este período, os pedaços cárneos foram enformados (fôrma de inox), prensadas e, submetidos à cocção a $353 \mathrm{~K}$ por 80 minutos. Em seguida, foram refrigerados a $277 \mathrm{~K}$ por 24 horas. Após este período, foram desenformados, acondicionados em sacos de polietileno de baixa densidade e mantidos sob refrigeração a $277 \mathrm{~K}$ até a realização das análises. Todas as análises foram realizadas

Tabela 1. Formulação dos presuntos

\begin{tabular}{|c|c|c|c|c|}
\hline Ingrediente & Controle (C) & F1 & F2 & F3 \\
\hline Pernil (\%) & 100 & 100 & 100 & 100 \\
\hline Sal (\%) & 2 & 1,7 & 1,4 & 1,1 \\
\hline $\begin{array}{c}\text { Cloreto de } \\
\text { Potássio (\%) }\end{array}$ & - & 0,3 & 0,6 & 0,9 \\
\hline
\end{tabular}

*A quantidade de conservante, estabilizante e antioxidante foram adicionado de acordo com a Resolução- RDC- ${ }^{\circ}$ 28, de fevereiro de 2001, normativa Brasileira.

em triplicata.

\section{ANÁLISES QUÍMICAS}

$\mathrm{O}$ teor de umidade foi determinado em estufa a vácuo (Tecnal, TE-395, Piracicaba, Brasil); o nitrogênio total com o método micro-Kjeldahl, em destilador de nitrogênio (Tecnal, TE-0363, Piracicaba, Brasil); os lipídeos em aparelho de Soxhlet (Tecnal, TE-044, Piracicaba, Brasil); as cinzas por incineração em mufla (EDG, Forno Economic, São Carlos, Brasil). Foram realizadas segundo as metodologias recomendadas pela AOAC (2012) $)^{9}$. O pH das amostras foi aferido utilizando-se potenciômentro (Hanna Instruments HI-9224). O teor de sódio foi determinado por fotometria de chama (Corning 400) conforme AOAC $(2005)^{10}$.

\section{ATIVIDADE DE ÁGUA}

A atividade de água foi medida à temperatura ambiente (298 $\mathrm{K} \pm 1$ ) usando um medidor de atividade de água Aqualab (Aqualab CX-2, Decagon Devices Inc., Pullman,
WA).

\section{CAPACIDADE DE RETENÇÃO DE ÁGUA (CRA)}

A mensuração da capacidade de retenção de água foi realizada nos presuntos após 24 horas de armazenamento a $278 \mathrm{~K}$, empregando-se a metodologia descrita por Houfmann et al., (1982) na qual amostras de 0,3 $\pm 0,005$ foram pesadas em papel filtro $\mathrm{n}^{\mathrm{o}} 2(100 \times 100 \mathrm{~mm}) \mathrm{e}$ posteriormente prensadas $\left(35 \mathrm{~kg} / \pm \mathrm{cm}^{2}\right)$ entre duas placas de vidro durante $5 \mathrm{~min}$, utilizando-se prensa manual hidráulica.

Decorrido o tempo, o conjunto foi desmontado e mediram-se as áreas das manchas deixadas pelo presunto

$$
C R A=\frac{A_{1}}{A_{2}} \times 100 \%
$$

e pela água exsudada. A capacidade de retenção de água foi calculada utilizando-se a equação 01, em que, CRA representa a capacidade de retenção de água, A1 a área da mancha deixada pela amostra e A2 a mancha deixada pela água exsudada da amostra ${ }^{11,12}$.

\section{ANÁLISE DE COR}

As determinações dos parâmetros instrumentais de cor $\left(\mathrm{L}^{*}, \mathrm{a}^{*} \mathrm{e} \mathrm{b*}\right.$ ) foram realizadas em colorímetro (Color Quest II, Hunter Lab Reston, Canadá), segundo método descrito por Paucar-Menacho et al. (2008) ${ }^{13}$. Todas as análises foram realizadas em triplicata.

\section{ANÁLISE MICROBIOLÓGICAS}

Contagem de Coliformes totais e termotolerantes a $318 \mathrm{~K}$, Clostridios sulfito redutores a $319 \mathrm{~K}$, assim como a pesquisa de presença de Salmonella sp. foram realizadas de acordo com metodologia descrita pela American Public Health Association (APHA, 2001) ${ }^{14}$.

\section{ANÁLISE SENSORIAL}


A análise sensorial dos presuntos foi realizada por 83 provadores não treinados pela Escola de Agronomia UFG, Goiânia, Goiás, Brasil. Foi realizado um teste de aceitação em escada hedônica de nove pontos (9 - gostei muitíssimo, 5 - indiferente, 1 - desgostei muitíssimo), avaliando os atributos cor, aparência, sabor e textura, das amostras padrão e com $15 \%, 30 \%$ e $45 \%$ de substituição do cloreto de sódio por cloreto de potássio.

\section{ANÁLISE ESTATÍSTICA}

$\mathrm{O}$ processamento e os dados instrumentais foram analisados utilizando o procedimento PROC MIXED da SAS (SAS Inst. Inc., Cary, NC). O modelo incluiu tanto os efeitos fixos (nível de cloreto de sódio, nível de cloreto de potássio) como aleatórios (replicação de processamento). O modelo para análise de dados sensoriais do consumidor incluiu adicionalmente o efeito aleatório do painelista. As médias de mínimos quadrados foram calculadas para o efeito principal da formulação e as médias foram separadas usando a opção de probabilidade de diferença com um ajuste de Tukey HSD quando os respectivos testes $\mathrm{F}$ foram significativos $(\mathrm{p} \leq 0,05)$.

\section{Resultados e Discussão}

\section{ANÁLISE CENTESIMAL}

$\mathrm{Na}$ Tabela 2, observa-se que não houve diferença significativa $(p>0.05)$ entre as formulações no teor de proteína. Pietrasik e Gaudette $(2014)^{7}$ obtiveram em seus estudos média de cerca $20.44 \%$ de teor de proteína ao substituir o cloreto de sódio por cloreto de potássio no desenvolvimento de presunto. $\mathrm{O}$ teor de proteína para o presunto é em média 22\%. Costa-Corredor et al. (2009) ${ }^{15}$ obtiveram cerca de $22.47 \%$. Os resultados obtidos neste estudo estão condizentes com os relatados na literatura. E são superiores ao valores encontrados Thomas et al. $(2008)^{16}$ que encontraram média de $18,6 \%$.

Em relação ao teor de lipídios, não houve diferença significativa $(p>0.05)$ entre as formulações. Os resultados obtidos foram semelhantes aos obtidos por Aaslyng et al. $(2014)^{3}$ no qual fizeram substituição de $2.5 \%$ e de $1.5 \%$ do cloreto de sódio por cloreto de potássio, apresentando respectivamente os seguintes valores, $2.23 \%, 2.84 \%$, e para o controle $3.37 \%$. Porém, os resultados obtidos neste estudo se diferiram dos obtidos por Pietrasik e Gaudette $(2014)^{7}$ que substituíram o cloreto de sódio em distintas proporções de sal sucademico (OF45, OF60, $\mathrm{SP}$ ), obtiveram em média $1.68 \%$ do teor lipídico. A diferença discrepante dos resultados obtidos neste estudo em relação aos da literatura podem ser explicados pelo seguinte fato, o teor de lipídios depende da espécie, idade, raça, sexo, nutrição, localização anatômica, qualidade da matéria-prima dentre outros fatores. Os autores Aaslyng et al. $(2014)^{3}$ usaram lombo de porco e Pietrasik e Gaudette $(2014)^{7}$ usaram músculo semimembranoso e adutor, neste foi usado pernil dessosado sem gordura. Observa-se também que houve uma variação no teor lipídico entre as formulações em estudo, variando de 3.06 a $1.93 \%$. Isto

Tabela 2. Propriedades químicas dos presuntos P (Padrão), F1 (15\% de substituição), F2 (30\% de substituição) e F3 (45\% de substituição).

\begin{tabular}{|c|c|c|c|c|c|}
\hline Formulação & $\begin{array}{l}\text { Proteínas } \\
\text { (\%/100 g) }\end{array}$ & $\begin{array}{l}\text { Lipídios } \\
\text { (\% /100 g) }\end{array}$ & $\begin{array}{c}\text { Cinzas } \\
(\% / 100 \mathrm{~g})\end{array}$ & $\begin{array}{c}\text { Carboidratos } \\
(\% / 100 \mathrm{~g})\end{array}$ & $\begin{array}{c}\text { Sódio } \\
\text { (mg / 100 g) }\end{array}$ \\
\hline $\mathrm{C}$ (Controle) & $22,27 \mathrm{a} \pm 0,77$ & $3,06 a \pm 0,32$ & $2,75 \mathrm{a} \pm 0,10$ & $0,31 \mathrm{a} \pm 0,03$ & $664,51 \mathrm{a} \pm 3,06$ \\
\hline F1 (15\%) & $22,35 \mathrm{a} \pm 0,24$ & $2,56 a \pm 0,02$ & $2,89 \mathrm{ab} \pm 0,07$ & $0,36 a \pm 0,02$ & $589,62 b \pm 2,69$ \\
\hline F2 (30\%) & $22,76 a \pm 1,76$ & $2,61 \mathrm{a} \pm 0,25$ & $3,17 \mathrm{ab} \pm 0,23$ & $0,31 \mathrm{a} \pm 0,02$ & $474,45 \mathrm{c} \pm 0,00$ \\
\hline F3 (45\%) & $21,59 a \pm 0,62$ & $1,93 a \pm 0,09$ & $3,30 b \pm 0,22$ & $0,70 b \pm 0,05$ & $359,16 \mathrm{~d} \pm 2,81$ \\
\hline
\end{tabular}

*Médias seguidas pela mesma letra, na mesma linha, não apresentaram diferença estatística significante no Teste de $\mathrm{F}$, a 5\%. Valores expressos em média seguidas pelo desvio padrão. 
provavelmente, se deve ao tipo de processo empregado no desenvolvimento do presunto em estudo, pois não foi realizada a etapa de tombamento em tumbler, pois esta tem por objetivo em uniformizar o pedaço cárneo com os ingredientes.

No atributo cinzas (Tabela 1), observa-se que não houve diferença significativa $(p>0.05)$ entre a formulação Controle e F1, porém diferiram significativamente das formulações F2 e F3, provavelmente isto pode ter sido ocasionado pelo aumento da concentração do cloreto de potássio e redução do cloreto de sódio. Os valores encontrados neste estudo foram próximo dos valores relatados na literatura por Abreu (2011). Horita et al. (2011)17 fizeram a substituição parcial do cloreto de sódio por cálcio de magnésio, obtiveram teor de cinzas médio cerca de $4.02 \%$.

O teor de carboidratos (Tabela 2) nas formulações controle, F1 e F2 não diferiram estatisticamente entre si $(\mathrm{p}>0.05)$. Porém, diferiram-se estatisticamente da formulação F3. De acordo com a USDA (2013) $)^{18}$, o teor médio de carboidratos deve ser de $0.69 \%$ em presuntos cozidos, a formulação $\mathrm{F} 3$ foi a única que apresentou tal valor, enquanto as demais formulações ficaram abaixo do proposto pela $\mathrm{USDA}^{18}$.

\section{TEOR DE SÓDIO}

Na Tabela 2 observa-se que houve diferença estatística $(\mathrm{p}<0.05)$ no teor de Sódio nas formulações. Conforme aumentou-se a concentração do cloreto de potássio e reduziu-se a concentração de cloreto de sódio, houve redução no teor de sódio presente nas formulações F1, F2 e F3 respectivamente, $11.31 \%, 28.50 \%$ e $45.95 \%$ em relação ao controle. Estes resultados são semelhantes aos obtidos por Costa-Corredor et al. (2009), Aaslyng et al. (2014) ${ }^{3}$, Pietrasik e Gaudete (2014) ${ }^{7}$, Greiff et al. $(2015)^{8}$ e Pietrasik et al $(2016)^{19}$ que obtiveram respectivamente, $8.36,34.6 \%$, $27.19 \%$ e $55 \%$. Os valores encontrados neste estudo estão semelhante aos dos autores mencionados, mas se deve ressaltar que as condições impostas neste foram distintas aos citados. Porém, os autores perceberam que à medida que se reduziu o cloreto de sódio, aumentou-se cloreto de potássio.
Segundo Desmond (2006) ${ }^{20}$ a redução do cloreto de sódio pode afetar diversas propriedades do presunto, uma vez que este é um ingrediente essencial em produtos cárneos. Influenciando o sabor, atividade de água, e consequentemente a vida de prateleira desses produtos. Além disto, uma das principais funções do sal em produtos cárneos é a solubilização das proteínas miofibrilares, isso ativa tais proteínas aumentando a hidratação e a capacidade de ligação com a água, melhorando, assim, a textura.

\section{PH E ACIDEZ TITULÁVEL}

Observa-se (Tabela 3) que o $\mathrm{pH}$ da formulação controle apresentou diferença estatística significativa $(\mathrm{p}<0.05)$ em relação aos demais tratamentos com cerca de $3.28 \%$. Estes resultados são similares aos obtidos por Horita et al. $(2011)^{17}$, Aaslyng et al. $(2014)^{3}$, Pietrasik e Gaudette $(2014)^{7}$, Greiff et al. $(2015)^{8}$ e Pietrasik et al. $(2016)^{19}$ que apresentaram respectivamente, $6.29,6.13,6.29,6.15 \mathrm{e}$ 6.26. Estes valores de $\mathrm{pH}$ são semelhantes aos valores de pH típico do presunto Norueguês (GEJAUTG-ENG et al., $2010)^{21}$. Observa-se também que conforme se aumentou a concentração do cloreto de potássio e reduziu-se o cloreto de sódio, o pH aumentou-se. Os autores Puolanne et al.

Tabela 3. Parâmetros químicos dos presuntos $\mathrm{P}$ (Padrão), F1 (15\% de substituição), F2 (30\% de substituição) e F3 (45\% de substituição).

\begin{tabular}{|c|c|c|}
\hline Formulação & $\mathbf{p H}$ & Acidez (\%) \\
\hline C (Controle) & $6.13 \mathrm{a} \pm 0.05$ & $0.72 \mathrm{ab} \pm 0.04$ \\
\hline F1 (15\%) & $6.30 \mathrm{~b} \pm 0.01$ & $0.65 \mathrm{a} \pm 0.01$ \\
\hline F2 (30\%) & $6.33 \mathrm{~b} \pm 0.01$ & $0.66 \mathrm{ab} \pm 0.04$ \\
\hline F3 (45\%) & $6.27 \mathrm{~b} \pm 0.04$ & $0.73 \mathrm{~b} \pm 0.03$ \\
\hline
\end{tabular}

*Médias seguidas pela mesma letra, na mesma linha, não apresentaram diferença estatística significante no Teste de F, a 5\%. Valores expressos em média seguidas pelo desvio padrão. 
(2001), Gjerlaung-Enger et al. (2010) ${ }^{21}$, Greiff et al. (2015) confirmaram isto também em seus estudos.

Para acidez, observa-se (Tabela 3) que a formulação F2 diferiu estatisticamente $(\mathrm{p}<0,05)$ do Controle, F1 e F3 cerca de $12.02 \%$. Estes resultados são inferiores aos apresentados por Thomas, Anjaneyulu e Kondaiah (2008)16 em seus estudos com o desenvolvimento de salsichas de porco com carne e a gordura quente, obtiveram acidez média de 1.25.

\section{ANÁLISE DE COR}

As formulação Controle (C) e F3 apresentaram coloração amarelada e enquanto as formulações F1 e F2 apresentaram coloração avermelhada. Porém, estatisticamente, não houve efeito da substituição do sal nos parâmetros instrumentais de cor (Tabela 3) analisados. Estes resultado estão de acordo com os de vários autores. Horita et al. (2011) ${ }^{17}$ relataram nenhum efeito na substituição de $50 \%$ do $\mathrm{NaCl}$ por $\mathrm{KCl}$ nos valores de L*, a* e b* no estudo da mortadela.

Conforme houve aumento na concentração de $\mathrm{KCl}$, houve aumento na luminosidade. Crehan, Troy, e Buckley $(2000)^{23}$ reduziu a concentração de $\mathrm{NaCl}$ de $2.5 \%$ para $1.5 \%$ em salsichas cozidas e não encontraram alterações nos valores de L*. Já Greiff et al. $(2015)^{8}$ encontraram valores distintos neste atributo em comparação com os valores obtidos neste estudo, quando substituíram o cloreto de sódio por cloreto de potássio em 14, 25, 35, 46 e 57\%, observaram que a redução do sal teve forte influência na tonalidade da cor.

Houve mudança na luminosidade, em função do teor de sal reduzido, com $25 \%$, apresentou maior luminosidade, nos demais tratamentos houve redução do atributo. Provavelmente isso pode ser explicado porque os autores Greiff et al. $(2015)^{8}$ embalaram os presuntos em atmosfera modificada, isto influenciou na coloração, pois os produtos em estudos foram acondicionados em sacos de polietinelo de baixa densidade sem modificação da atmosfera. Porém, Aaslyng et al. (2014) mencionam que a cor dos produtos curados é menos afetado pela pressão, em comparação com produtos de carne crua. Resistência do pigmento nitrosilmioglobina a oxidação
Tabela 4. Efeito de cor dos presuntos P (Padrão), F1 (15\% de substituição), F2 (30\% de substituição) e F3 (45\% de substituição).

\begin{tabular}{|c|c|c|c|}
\hline Formulação & $\mathbf{L}^{*}$ & $\mathbf{a}^{*}$ & $\mathbf{b}^{*}$ \\
\hline $\mathrm{C}($ Controle $)$ & $62.07 \mathrm{a} \pm 2.62$ & $5.43 \mathrm{a} \pm 0.92$ & $5.79 \mathrm{a} \pm 0.25$ \\
\hline $\mathrm{F} 1(15 \%)$ & $52.84 \mathrm{a} \pm 2.48$ & $6.75 \mathrm{a} \pm 0.65$ & $3.8 \mathrm{a} \pm 0.36$ \\
\hline F2 $(30 \%)$ & $58.65 \mathrm{a} \pm 4.06$ & $5.49 \mathrm{a} \pm 0.69$ & $3.83 \mathrm{a} \pm 0.89$ \\
\hline F3 (45\%) & $59.02 \mathrm{a} \pm 0.19$ & $3.8 \mathrm{a} \pm 1.35$ & $4.13 \mathrm{a} \pm 0.89$ \\
\hline
\end{tabular}

*Médias seguidas pela mesma letra, na mesma linha, não apresentaram diferença estatística significante no Teste de F, a 5\%. Valores expressos em média seguidas pelo desvio padrão.

é provavelmente a razão para a estabilidade de cor de produtos de charcutaria ${ }^{3}$.

\section{UMIDADE}

O teor de umidade (Tabela 5) das formulações não apresentaram diferenças significativas entre si $(p<0.05)$. Porém, observou-se que com a redução da concentração do cloreto (F3) de sódio houve aumento da umidade. Os valores reportados neste trabalho estão em conformidade com os estudos de Aaslyng et al. (2014) $)^{3}$, Everts et al. (2010) ${ }^{24}$, que obtiveram respectivamente $73.96 \%$ e $74.77 \%$ do teor médio de umidade em presuntos sem a substituição do cloreto de sódio. Pietrasik e Gaudette $(2014)^{7}$ produziram presuntos substituindo $30 \%$ do cloreto de sódio e obtiveram umidade em torno de $74.4 \%$. Greiff et all. $(2015)^{8}$ perceberam que quanto maior a percentagem de substituição do cloreto de

Tabela 5. Parâmetros químicos dos presuntos $\mathrm{P}$ (Padrão), F1 (15\% de substituição), F2 (30\% de substituição) e F3 (45\% de substituição).

\begin{tabular}{|c|c|c|c|}
\hline Formulação & Umidade (\%) & Aw & CRA (\%) \\
\hline P (Padrão) & $71.60 \mathrm{a} \pm 1.28$ & $0.974 \mathrm{a} \pm 0.005$ & $96.00 \mathrm{a} \pm 1.52$ \\
\hline F1 (15\%) & $71.84 \mathrm{a} \pm 1.20$ & $0.973 \mathrm{a} \pm 0.001$ & $88.06 \mathrm{a} \pm 5.03$ \\
\hline F2 (30\%) & $71.15 \mathrm{a} \pm 1.50$ & $0.973 \mathrm{a} \pm 0.004$ & $87.94 \mathrm{a} \pm 4.11$ \\
\hline F3 (45\%) & $72.48 \mathrm{a} \pm 0.26$ & $0.980 \mathrm{a} \pm 0.001$ & $75.84 \mathrm{~b} \pm 1.17$ \\
\hline
\end{tabular}

*Médias seguidas pela mesma letra, na mesma linha, não apresentaram diferença estatística significante no Teste de F, a 5\%. Valores expressos em média seguidas pelo desvio padrão. 
potássio mais elevada foi a umidade. O tratamento Controle apresentou teor de umidade de $74.50 \%$ enquanto com $57 \%$ de substituição foi de $75.40 \%$.

Armenteros et al. (2012) ${ }^{1}$, em seus estudos, substituíram o cloreto de sódio por cloreto de potássio e cloreto de magnésio (I controle ( $\mathrm{NaCl}(100 \%))$; II: $\mathrm{NaCl}(50 \%)$ e $\mathrm{KCl}(50 \%)$; III : $\mathrm{NaCl}(55 \%), \mathrm{KCl}(25 \%)$ e $\mathrm{CaCl} 2(15 \%)$ e $\mathrm{MgCl} 2$ realizaram a evolução da umidade ao longo do período de armazenamento por 270 dias e observou-se que houve uma diminuição significativa do teor de umidade. Isso pode ser explicado ao longo do período de estocagem o sal penetra no pedaço cárneo, reduzindo, assim, o teor de umidade presente. Inicialmente, apresentou-se cerca de $70.17 \%$ e no final da estocagem, com $52.47 \%$.

\section{ATIVIDADE DE ÁGUA (AW)}

Para a atividade de água (Tabela 5), não houve diferença significativa entre os tratamentos $(p>0.05)$, quando ao fazer a substituição do cloreto de sódio por cloreto de potássio. Estes resultados são semelhantes aos obtidos por Pietresaki e Gaudette (2014) ${ }^{7}$ em torno de 0.979 e Greiff et al. $(2015)^{8}$ obtiveram 0.965. Mossel e Corry (1995)25 relatam que tais níveis de aw em presuntos cozidos proporciona a deterioração de microrganismos patogênicos indesejáveis nestes tipos de alimentos.

\section{ANÁLISE DE COR}

Observa-se, na Tabela 5, que a Capacidade de Retenção de Água (CRA) da formulação F3 se diferiu estatisticamente $(\mathrm{p}<0.05)$ das outras formulações (Padrão, F1 e F2). Como esperado, observou-se uma diminuição da CRA nas formulações, conforme aumentou-se a concentração do cloreto de potássio e reduziu-se o cloreto de sódio. Os resultados apresentados neste estudo estão em conformidade com Greif et al. (2015) que observaram isso também em suas pesquisas, nas quais o teor de sal foi reduzido em $60 \%$ (NaK40) e (NaK20) (em base molar), correspondente a um teor de sal cerca de $1.73 \%$ e $1.38 \%$, respectivamente. Ramírez et al. (2002) $)^{26}$,em seus estudos, confirmaram isto ao avaliarem a capacidade de retenção de água em presuntos cozidos formulados com $0,1 \%$ e $2 \%$ de cloreto de sódio, verificaram que, à medida que se reduz o teor de cloreto de sódio na formulação, ocorre uma diminuição na capacidade de retenção de água. Provavelmente, a redução da CRA ocorreu devido à menor capacidade de extração de proteínas miofibrilares do cloreto de potássio comparado ao cloreto de sódio. Albarracín e Sánchez $(2011)^{27}$ explicaram que o aumento da CRA em carne é resultante do aumento do teor de sal, é devido aos ânion preferencialmente se ligam nas moléculas da proteína. Ham $(1972)^{28}$ descobriu que o cloreto de sódio aumenta a capacidade de retenção da água ligada na carne.

\section{ANÁLISE SENSORIAL}

Não houve diferenças significativas $(p>0.05)$ na substituição do cloreto de sódio por cloreto de potássio entre todas as formulações quanto à aparência, textura, sabor e odor (Tabela 6). O sabor é considerado um atributo crucial para as formulações de presuntos, observa-se que, nas concentrações $45 \%$ e $30 \%$ de cloreto de potássio, houve rejeição por parte dos provadores. Horita et al. $(2011)^{17} \mathrm{e}$ Pietresaki et al. (2016) ${ }^{19}$ obtiveram resultados semelhantes neste atributo, com média de 6 e 6,21, respectivamente. Totosaus et al. $(2004)^{29}$ realizaram substituição parcial do cloreto de sódio por $\mathrm{CaCl}_{2}$ em produtos emulsionados usando k-carragena e não encontraram diferenças sensoriais em comparação com a amostra controle. Horita et al. $(2011)^{17}$ e Guàrdia et al. (2006) $)^{30}$ constataram que houve diferenças perceptíveis para o atributo sabor, afirmam que a rejeição pode ter sido causada pelo sabor amargo conferido pelo cloreto de potássio ao produto.

O cloreto de potássio é o substituto mais utilizado na preparação de produtos à base de carne de baixo teor de sódio, mas, como observado no presente estudo, dependendo do nível de substituição, que resulta em rejeição sensorial porque produz um sabor amargo e metálico ${ }^{31,32}$. No estudo de Guàrdia et al. $(2006)^{30}$, um gosto amargo foi detectado em embutidos fermentados contendo cloreto de sódio substituído por $\mathrm{KCl}$, que foi aceito a uma substituição de $40 \%$. De acordo com relatórios anteriores, o sabor amargo foi o atributo mais perceptível em produtos cárneos fermentados com substituição parcial do $\mathrm{NaCl}$ por $\mathrm{KCl}^{33,34}$. O painel de provadores treinados no estudo 
Tabela 6. Atributos sensoriais dos presuntos P (Padrão), F1 (15\% de substituição), F2 (30\% de substituição) e F3 (45\% de substituição).

\begin{tabular}{|c|c|c|c|c|}
\hline Formulação & Sabor & Textura & Aparência & Odor \\
\hline C (Controle) & $6.78 \mathrm{a} \pm 1.91$ & $6.48 \mathrm{a} \pm 1.71$ & $6.89 \mathrm{a} \pm 1.49$ & $6.42 \mathrm{a} \pm 1.63$ \\
\hline F1 $(15 \%)$ & $7.08 \mathrm{a} \pm 1.66$ & $6.82 \mathrm{a} \pm 1.50$ & $6.96 \mathrm{a} \pm 1.66$ & $6.49 \mathrm{a} \pm 1.91$ \\
\hline F2 $(30 \%)$ & $6.64 \mathrm{a} \pm 1.94$ & $6.80 \mathrm{a} \pm 1.65$ & $6.64 \mathrm{a} \pm 1.95$ & $6.19 \mathrm{a} \pm 1.85$ \\
\hline F3 $(45 \%)$ & $6.59 \mathrm{a} \pm 1.80$ & $6.69 \mathrm{a} \pm 1.70$ & $6.73 \mathrm{a} \pm 1.69$ & $6.34 \mathrm{a} \pm 1.64$ \\
\hline
\end{tabular}

*Médias seguidas pela mesma letra, na mesma linha, não apresentaram diferença estatística significante no Teste de F, a 5\%. Valores expressos em média seguidas pelo desvio padrão.

de Zanardi et al. (2010) ${ }^{34}$, também detectada uma diminuição do sabor salgado e uma cor mais clara em amostras de salame baixo teor de sódio, resultando em menor aceitabilidade global em comparação com o controle.

Em relação à textura, as formulações $\mathrm{F} 1 \mathrm{e} \mathrm{F} 2$ foram as que tiveram maior aceitação, pois com $15 \%$ e $30 \%$ substituição não altera a textura perceptivelmente ao consumidor. Horita et al. (2011) $)^{17}$ e Prietraski et al. (2016) ${ }^{19}$ observaram que a substituição do cloreto de sódio por cloreto de potássio não afetou o atributo textura. Isto provavelmente deve ter ocorrido pois o cloreto de potássio e cloreto de sódio têm a mesma propriedade de solubilização das proteínas miofibrilares, isto ativa tais proteínas aumentando, assim, a hidratação e a capacidade de ligação com a água, melhorando assim a textura ${ }^{20}$.

Para o atributo aparência, as formulações F1 e Controle tiveram maior aceitação. Pietrasik e Gaudette (2014)7 avaliaram o impacto da substituição do cloreto de sódio por cloreto de potássio, e intensificador de sabor sobre as características de processamento e aceitação pelo consumidor de presunto cozido reestruturado. E relatam que quanto maior for a concentração de $\mathrm{KCl}$ maior será a luminosidade, ou seja, em concentrações o $\mathrm{KCl}$ afeta a aparência do produto, isto foi observado neste estudo.

\section{ANÁLISE MICROBIOLÓGICA}

Na Tabela 7, observam-se os resultados das contagens de coliformes a $45{ }^{\circ} \mathrm{C}$, Staphylococcus coagulase positiva, Clostridium sulfito redutores e pesquisa de Salmonella para as quatro formulações de presunto produzidas. Todas as contagens foram abaixo dos limites aceitáveis para alimentos pronto para serem consumidos e estão abaixo dos valores estabelecidos pela RDC 12 $\left(\right.$ BRASIL, 2001) ${ }^{35}$. Dessa forma, ficam evidenciadas

Tabela 7. Avaliação microbiológica dos presuntos P, F1, F2 e F P (Padrão), F1 (15\% de substituição), F2 (30\% de substituição) e F3 (45\% de substituição).

\begin{tabular}{|c|c|c|c|c|c|}
\hline & \multicolumn{4}{|c|}{ Formulação } & \multirow{2}{*}{$\begin{array}{c}\text { Padrões RDC } 12 \mathrm{p} / \\
\text { Presunto }\end{array}$} \\
\hline & $\mathrm{P}$ & F1 & $\mathrm{F} 2$ & F3 & \\
\hline Coliformes a $45^{\circ} \mathrm{C}(\mathrm{UFC} / \mathrm{g})$ & $<100$ & $<100$ & $<100$ & $<100$ & $1.0 \times 10^{3}$ \\
\hline Estafilococos coagulase positiva (UFC/g) & $<100$ & $<100$ & $<100$ & $<100$ & $3.0 \times 10^{3}$ \\
\hline Clostridios sulfito redutores a $46^{\circ} \mathrm{C}(\mathrm{UFC} / \mathrm{g})$ & $<100$ & $<100$ & $<100$ & $<100$ & $5.0 \times 10^{2}$ \\
\hline Salmonella sp/25 g & Aus. & Aus. & Aus. & Aus. & Aus. \\
\hline
\end{tabular}

*Aus.= Ausência 
as condições higiênicas e sanitárias, assim, como, as boas práticas de fabricação e manipulação adotadas no processamento dos presuntos, tornando-os aptos ao consumo humano.

Além disso, a substituição parcial de cloreto de sódio por cloreto de potássio, em até $45 \%$, não influenciou na microbiota presunto, inicialmente. Estes resultados estão de acordo com os obtidos por Pietrasik e Gaudette (2014) ${ }^{7}$ avaliaram o impacto dos substitutos do sal e do intensificador de sabor sobre as características de processamento e aceitação do consumidor de presuntos cozidos reestruturados, não encontram influência dos substitutos do sal no crescimento dos microrganismos. Aaslyng et al. $(2014)^{3}$ avaliaram a influência da redução parcial de sal por cloreto de potássio em salame, salsicha, bacon e presunto não encontrando diferenças no crescimento microbiano a não ser leveduras em bacon. De forma semelhante, Blesa et al. $(2008)^{36}$ também estudou o efeito da substituição parcial de cloreto de sódio por outros sais e não encontrou diferença significativa entre as formulações em relação à contagem microbiana.

\section{Conclusão}

A composição centesimal da formulação com $45 \%$ de substituição do cloreto de sódio por cloreto de potássio aumentou o teor de cinzas e carboidratos. $\mathrm{Na}$ avaliação microbiológica, não foi influenciada pela substituição do cloreto de sódio por cloreto de potássio. Com relação às características físico-químicas, atividade de água e cor não foram afetadas. $\mathrm{O} \mathrm{pH}$ e a capacidade de retenção de água das formulações foram afetados. À medida em que se aumentou o nível de substituição do cloreto de sódio por cloreto de potássio, diminuiu-se a capacidade de água e o $\mathrm{pH}$. Entretanto, tais alterações nas características não afetaram a aceitabilidade dos presuntos pelo consumidor. Portanto, a substituição parcial do cloreto de sódio por cloreto de potássio em até $45 \%$ é completamente viável em presuntos sem grande prejuízo às características físico-químicas e sensoriais do produto, tendo melhor aceitabilidade por parte do consumidor ao nível de $15 \%$ de substituição.

\section{Referência Bibliográfica}

1. Armenteros, M.; Aristoy, M.; Barat, J.; Toldrá, F. Biochemical and sensory changes in dry-cured ham salted with partial replacements of $\mathrm{NaCl}$ by other choride salts. Meat Science, 90, 361-367, 2012.

2. Bibbins-Domingo, G.M.; Chertow, P.G.; Coxson, A.; Moran, J.M.; Lightwood, M.J.; Pletcher, L. Projected effect of dietary salt reductions on future cardiovascular disease. The New England Journal of Medicine, 362, 590-599, 2010.

3. Aaslyng. ; M. D.; Vestergaard, C.; Koch, A. G; Koch, A. G. The effect of salt reduction on sensory quality and microbial growth in hotdog sausages, bacon, ham and salami. Meat Science, 96,1, 47-55, 2014.

4. Ni, M.C.; Capelin, E.K.; Duford, J.L.; Webster, B.C.; Neal, S.A. Sodium contente of processed foods in the United Kingdom: analysis of 44,000 foods purchased by 21,000 households. American Journl of Clinical Nutrition, 93, 594-600, 2010.

5. Webster, L. Webster, E.K., Dunford, B.C. A systematic survey of the sodium contents of processed foods. American Journal of Clinical Nutrition, 91, 413-420, 2010.

6. Verma, A.; Banerjef, R.. Low-Sodium Meat Products: Retaining Salty Taste for Sweet Health. Critical Reviews in Food Science and Nutrition, 52, 72-84, 2012.

7. Pietrasick, Z.; Gaudette, N. The impact of salt replacers and flavor enhancer on the processing characteristics and consumer acceptance of restructured cooked hams. Meat Science., 96, 1165-1170, 2014.

8. Greiff, K.; Mathiassaen, J.R.; Misimi, E.; Hersielth, M.; Ausand, I.G. Gradual reduction in Sodium Content in Cooked ham, with corresponding change in Sensorial Properties Measured by Sensory Evaluation and a Multimodal Machine Vision Sysem. POLS ONE, $1-15,2015$.

9. AOAC . Association of Official Analytical Chemists. Official Methods of Analysis. 19. ed. Washington, D.C.: AOAC, 2012.

10. AOAC. Association of Analytical Communities. AOAC official method. Sodium and potassium in seafood. Flame photometric method. Official Méthods of Analysis. 969.23. AOAC, 2005.

11. Grau, R. Fleisch und Fleischwaren., Verlag A.W. Hayn's Erben, ed. $1,1972$.

12. Hoffmann, K.; Hamm, R (1992) Neues über die Bestimmung der Wasser bindung des Fleisches mit Hilfe der Filterpapierpressmethode. Fleischwirtschaft, 62, 1, 87-94.

13. Paucar-Menacho, L. M.; Silva, L. H., Barretto, P. A. A., Mazal, G.; Fakhouri, F. M., Steel, C. J., \& Collares-Queiroz, F. P. Development of functional fresh pasta with the addition of soy protein isolate and polydextrose using paprika as a dye. Food Science and Technology, 28, 4, 2008. 
14. American Public Health Association. Compendium of Methods for the Microbiological Examination of foods. APHA, 4.ed., 2011.

15. Costa-Corredo. R.; Bergamin, W. F.; Cipolli, K. M. V. A. B.; Silveira, E. T. F.; Felício, P. E. Sensory profile and acceptance of raw hams produced by traditional methods and accelerated. Food Science and Tecnhology, 27 (1), 170-176, 2007.

16. Thomas, R.; Anjaneylu, A.S.R; Kondaiah, N. Effect of hot-boned pork on the quality of hurdle treated porl sausages during ambiente temperature $\left(37 \pm 1^{\circ} \mathrm{C}\right)$ storage. Food Chemistry, 107, 804-812, 2008.

17. Horita, C. N.; Morgano, M.; Celeghini, R. M. S.; Pollonio, M. A. R. Physico-chemical and sensory properties of reduced-fat mortadella prepared with blends of calcium, magnesium and potassium chloride the partial substitutes for sodium chloride. Meat Science, 89, 426433, 2011.

18. USDA. In: National Nutrient Database for Standard Reference Release 26 United States Department of Agriculture (2013) Available at: $<$ http://www.ars.usda.gov/Services/docs.htm?docid=8964> Access on July 15, 2014

19. Pietrasik, Z.; Gaudette, N.J.; Johnston, S.P The use of high pressure processing to enhance the quality and shelf life of reduced cooked hams. Meat Science, 116, 102-109, 2016.

20. Desmond, E.. Reducing salt: a challenge for the meat industry. Meat Science, 74, 188-196, 2006.

21. Gerjlaug-Enger E.; Aas L.ødegård J.; Vangen O. Genetic parameters of meat quality traits in two pig breeds measured by rapid methods. Animal, 11, 1832-1843, 2010.

22. Puolanne, E.; Ruusunen, M.H.; Vainionpää, J.i. Combined effects of $\mathrm{NaCl}$ and raw meat $\mathrm{pH}$ on water-holding in cooked sausage with and without added phosphate. Meat Science, 1, 581-587, 2001.

23. Crehan, C.m. ; Hughes, E. ; Troy, D.J. ; Buckey, D.J. Effects of fat level and maltodextrin on the functional properties of frankfurters formulated with 5, 12 and 30\% fat. Meat Science, 55 , p. 463-469, 2000.

24. Everts, A.j.; Wulf D.m.; Nath T.m.; Jennings, T.d.; Weaver, L. Quality characteristics of chunked and formed ham from pale average and dark muscles were improved using na ammonium hydroxide curing solution. Meat Science, 86, 353-356, 2007.

25. Mossel, D.A.A. ; Corry, J.E.T. ; Struijk, C.B. ; Bair, R.M. Essentials of the microbiology of foods. A Textbook for Advanced Studies: John Wiley \& Sons, Chichester, England; 1995.

26. Ramírez, J. Using salt and microbial transglutaminase as binding agentes in restructured fish products resembling hams. Food Science, 67 (5), 1778-1784, 2002.

27. Albarracín, W.; Sánchez, I.C; Grau, R.; Barat, J.M. Salt in food processing usage and reduction: are view. Food Science Technology, 46 (7), 1329-1336, 2011.

28. Hamm, R. Koloid chemieds. Fleisches: Parey, 1972.
29. Totosaus, A.; Alfaro-Rodrigues, R.H.; Pérez-Chabela, M.L. Fat and sodium chloride in sausages using k-carrageenan and other salts. International Journal of Food Sciences and Nutrition, 55 (5),371380, 2004.

30. Guàrdia, M. D.; Guerrero, L.; Gelabert, J.; Gou, P.; Arnau, J. Consumer attitude towards sodium reduction in meat products and acceptability of fermented sausages with reduced sodium content. Meat Science, 73, p. 484-490, 2006.

31. Guàrdia, M. D.; Guerrero, L.; Gelabert, J.; Gou, P.; Arnau, J. Sensory characterisation and consumer acceptability of small calibre fermented sausages with $50 \%$ substitution of $\mathrm{NaCl}$ by mixtures of $\mathrm{KCl}$ and potassium lactate. Meat Science, 80, p. 1225-1230, 2008

32. Seman, D.L; Olson, D.G.; Mandigo, R.W. Effect of reduction and partial replacement of sodium on bologna characteristics and acceptability. Journal of Food Science, 45, 1116-1121, 1980.

33. Gou, J.; Arnau H.; Guerrero, L. The influence of meat pH on mechanical and sensory textural properties of dry-cured ham. Meat Science, 52, 267-273, 1999.

34. Zanardi E., Ghidini S., Conter M.; \& Ianieri A. Mineral composition of Italian salami and effect of $\mathrm{NaCl}$ partial replacement on compositional, physico-chemical and sensory parameters. Meat Science, 3, 86, 742-747, 2010 .

35. Brazil, National Health Surveillance Agency. RDC Resolution No. 12 of 02 January 2001. Approves the Technical Regulation on microbiological standards for food. Brasília, DF: ANVISA, 2001. Available at: $<$ http://www.anvisa.gov.br/e-legis/>. Accessed on: 15 October 2016

36. Blesa, E.; Sliño, M.; Barat, J. M.; Brau, R.; Toldrá, F.; Pagán, M. J. .Microbiology and physicochemical changes of dry-cured ham during the post-salting stage as affected by partial replacement of $\mathrm{NaCl}$ by other salts. Meat Science, 78, 135-142, 2008.
Universidade Federal de Goiás- UFG Campus Samambaia, Rodovia Goiânia-Nova Veneza, Km 0- Caixa Postal 131, CEP 74690- 900, Goiânia, Brasil.

*E-mail: julievanyufg@gmail.com 